\title{
PERCEPÇÃO DOS FAMILIARES DE PACIENTES COM ALERGIA AO LEITE DE VACA EM RELAÇÃO AO TRATAMENTO
}

\author{
PERCEPTIONS OF CAREGIVERS OF PATIENTS WITH COW'S MILK \\ ALLERGY REGARDING THE TREATMENT
}

\author{
Glauce Hiromi Yonamine ${ }^{1}$, Divanice Contim², Ana Paula Beltran Moschione Castro³, \\ Cristina Miuki Abe Jacob ${ }^{4}$, Antonio Carlos Pastorino 5
}

\begin{abstract}
Resumo:
Este estudo qualitativo teve como objetivo compreender as percepções de familiares de crianças e adolescentes com alergia à proteína do leite de vaca (APLV) em relação à doença e seu tratamento. Foram realizadas nove entrevistas e foi utilizado o método de análise de conteúdo. Surgiram três categorias com subcategorias: tratamento e educação do paciente e familiares (experiências vividas, base do tratamento e como lidar com a doença), resolução da doença (expectativa e melhora gradativa), qualidade de vida (inclusão social, cotidiano familiar e custo dos alimentos). Os familiares vivenciaram dificuldades durante o início do tratamento, mas revelaram que as orientações fornecidas no seguimento tornaram as adaptações à doença mais fáceis. Comentaram sobre as dificuldades em obter a colaboração de outros membros da família em relação à dieta de exclusão, suas experiências frente a uma reação alérgica, dúvidas quanto ao tratamento e lacunas do conhecimento sobre a doença entre outros médicos e na população em geral. Alguns deles acreditavam que não havia tratamento para a APLV, porque não existiam medicamentos ou vacinas, mas mantinham a esperança da descoberta de uma cura. A maioria dos familiares estava satisfeita com a melhora gradativa dos seus filhos, percebida pela redução da gravidade dos sintomas e tolerância a traços de leite. Também comentaram sobre os esforços em proporcionar uma vida normal para seus filhos, as mudanças em suas vidas e a dificuldade em comprar alimentos especiais. Em conclusão, os familiares de crianças e adolescentes com APLV sentem grande impacto da doença.
\end{abstract}

Palavras-chave: pesquisa qualitativa; hipersensibilidade alimentar; criança; hipersensibilidade a leite.

\begin{abstract}
:
The aim of this qualitative study was to understand the perceptions of caregivers of children and adolescents with cow's milk allergy regarding the disease and its treatment. Nine caregivers were interviewed and content analysis method was used. Three categories with subcategories emerged: treatment and education of the patient and their caregivers (life experiences, bases of treatment, coping with the disease), resolution of the disease (hope, gradual improvement), quality of life (social inclusion, family daily activities, costs of dietary treatment). Caregivers experienced difficulties during the initial treatment but pointed out that the guidance given during follow-up made the adjustments easier. Family members commented on the difficulties about lack of cooperation from other family members regarding the restrictive diet, their experience coping with the allergic reaction, doubts about the treatment and gaps on knowledge about the disease by other physicians and people. Some of them believed that there is no treatment for the disease, because there are no drugs or vaccines, but they were waiting for cure. The majority of relatives were satisfied with the gradual improvement of patients observed by reduction on the severity of symptoms and tolerance of milk traces within foods. In addition, they commented on the efforts to give a normal life for their children, the changes in their daily lives and the difficulty to buy special products. Concluding, caregivers of children and adolescents with cow's milk allergy feel a great burden of the disease.
\end{abstract}

Key word: qualitative research; food hypersensitivity; child; milk hypersensitivity.

1 Nutricionista, Mestre em Ciências, Supervisora do Ambulatório do Serviço de Nutrição e Dietética do Instituto da Criança do Hospital das Clínicas da Faculdade de Medicina da Universidade de São Paulo (ICr-HCFMUSP), Brasil.

2 Enfermeira, Doutora em Enfermagem, Professora Adjunta da Universidade Federal do Triângulo Mineiro, Uberaba, Brasil.

3 Médica, Doutora em Ciências, Assistente da Unidade de Alergia e Imunologia (ICr-HCFMUSP), Brasil.

4 Médica, Doutora em Ciências, Professora Associada do Departamento de Pediatria da FMUSP, Chefe da Unidade de Alergia e Imunologia (ICr-HCFMUSP), Brasil.

5 Médico, Doutor em Ciências, Assistente da Unidade de Alergia e Imunologia, (ICr-HCFMUSP), Brasil.

Instituição em que o trabalho foi realizado: Departamento de Pediatria - Unidade de Alergia e Imunologia. Instituto da Criança do Hospital das Clínicas de São Paulo - FMUSP, (ICr-HCFMUSP), Brasil.

Corresponding author: glaucehy@uol.com.br

Suggested citation: Yonamine $\mathrm{GH}$, et al. Perceptions of caregivers of patients with cow's milk allergy regarding the treatment; Journal of Human Growth and Development 2013; 23(1): 58-64

Manuscript submitted Oct 30 2012, accepted for publication Dec 282012. 


\section{INTRODUÇÃO}

Estudos demonstram que as alergias alimentares (AAs) afetam negativamente a qualidade de vida (QV) dos pacientes e familiares, especialmente por ser uma doença onde a restrição alimentar é a principal forma de tratamento, com a necessidade constante de vigilância para as reações alérgicas. Os profissionais de saúde exercem papel importante na melhoria da QV desta população, fornecendo informações sobre a doença e substitutos adequados nutricionalmente ${ }^{1}$.

Em recente revisão da literatura, os autores avaliaram o impacto psicossocial da alergia alimen$\operatorname{tar}(A A)$ e hipersensibilidade alimentar em crianças, adolescentes e seus familiares. Constatou-se que a QV de alguns subgrupos de pacientes e seus cuidadores parece ser mais negativamente afetada, especialmente entre pacientes do gênero feminino, com maior número de AAs, grande número de reações alérgicas prévias ou outras doenças atópicas associadas ${ }^{1}$.

Além disso, há evidências de que as crianças com AA que apresentaram anafilaxia sofrem mais do que os portadores de outras doenças crônicas, como doenças reumatológicas ou diabetes mellitus insulino dependente. Este fato parece estar relacionado com o medo constante de uma possível reação anafilática e consequente risco de morte, gerando alto nível de ansiedade e estresse para o paciente e sua família².

Apesar da importância do tema, ainda existem poucos estudos neste sentido, sendo que a maioria aborda grupos de pacientes com alta prevalência de alergia a amendoim e/ou frutas oleaginosas ${ }^{3-9}$. Por outro lado, em nosso país, o leite de vaca é um dos principais alérgenos nesta faixa etária e sua exclusão é extremamente difícil, por sua ampla distribuição em produtos alimentícios e sua importância nutricional na alimentação da criança e mesmo assim, não se encontram trabaIhos onde se abordem os problemas relacionados a essa restrição.

Assim, o objetivo é compreender as percepções de familiares de crianças e adolescentes com alergia à proteína do leite de vaca (APLV) em relação à doença e seu tratamento.

\section{MÉTODO}

Foi utilizada a abordagem qualitativa, pois permite avaliar a subjetividade e enfatiza a compreensão dos processos e fenômenos vivenciados (percepções, motivações, atitudes, valores, crenças e tendências) $)^{10,11}$.

Este estudo foi realizado no ambulatório de AA da Unidade de Alergia e Imunologia do Instituto da Criança do Hospital das Clínicas da Faculdade de Medicina da Universidade de São Paulo (ICrHCFMUSP), no período de Outubro de 2009 a Janeiro de 2010.
O estudo foi aprovado pela Comissão de Ética para Análise de Projetos de Pesquisa da Diretoria Clínica do Hospital das Clínicas e da Faculdade de Medicina da Universidade de São Paulo sob o número 513/09 e a pesquisa foi conduzida de acordo com os padrões éticos exigidos. Os participantes foram esclarecidos acerca do trabalho e assinaram o Termo de Consentimento Livre e Esclarecido.

A amostra foi constituída por familiares diretamente envolvidos nos cuidados de crianças e adolescentes (até 19 anos) com APLV, mediada pela Imunoglobulina $\mathrm{E}$ (IgE), com ou sem manifestações de anafilaxia, com acompanhamento mínimo de 1 ano. O diagnóstico de APLV foi realizado por meio de exames laboratoriais (teste cutâneo de hipersensibilidade imediata e/ou RAST/ ImmunoCAP) e/ou teste de provocação oral. Foram excluídos os familiares ou acompanhantes de crianças e adolescentes com APLV que não eram diretamente responsáveis por elas ou de pacientes que apresentavam, além da APLV, outras doenças crônicas não alérgicas que afetassem suas atividades diárias.

Entre os familiares elegíveis, foi selecionado aquele que compareceu primeiramente no dia do atendimento, sendo coletada uma entrevista por dia, antes da consulta médica, para que não houvesse interferência do que havia sido discutido durante $o$ atendimento.

Dados dos pacientes foram coletados dos registros dos prontuários e as características dos entrevistados foram obtidas no início da entrevista, de acordo com um roteiro semi-estruturado. Todos os nomes foram substituídos por nomes fictícios.

Foram realizadas entrevistas, utilizando-se gravador digital, propondo-se para que os participantes falassem livremente, a partir das seguintes perguntas orientadoras: "Fale sobre a sua experiência com o tratamento da APLV" e "O que o(a) $\operatorname{Sr}(\mathrm{a})$ espera do tratamento da doença do seu(sua) filho(a)?"

Os depoimentos foram coletados em um local previamente selecionado e sem horário previamente acordado. Não foram realizados comentários ou outras perguntas por parte da pesquisadora.

As entrevistas foram transcritas e transcriadas, como possibilidade de superar a mera aquisição de dados em favor da possibilidade de uma visão mais subjetiva das vivências depoentes $^{12}$.

Os depoimentos foram analisados pelo método de análise de conteúdo ${ }^{13}$. Este método é largamente utilizado e avalia profundamente a fala do entrevistado, para que, após uma observação cuidadosa, se possa compreender criticamente o sentido de sua mensagem, seja ele explícito, oculto ou obscuro ${ }^{14}$.

Primeiramente, foi realizada a identificação das unidades de significado, pela leitura flutuante 
e seleção das ideias principais de cada depoimento (palavras, frases, expressões ou trechos). Foram representadas por códigos, de acordo com o número do Discurso (letra $D$, seguida do número) e aparecimento da Unidade de Significado no texto (letra US, seguida de número). Em seguida, foram utilizados dicionários ortográficos da língua portuguesa ${ }^{15,16}$ para auxiliar na interpretação de cada unidade de significado ${ }^{13}$.

As unidades de significado foram classificadas segundo suas semelhanças e diferenças e foram definidas categorias, as quais foram analisadas. A determinação do número de categorias e suas denominações foram estabelecidas pelos pesquisadores e conferidas por um terceiro pesquisador externo.

\section{RESULTADOS}

Foram entrevistados nove familiares de crianças e adolescentes com APLV. Todos eram do gênero feminino, sendo uma delas avó da criança e principal cuidadora. A idade das entrevistadas variou de 25 a 58 anos e a escolaridade variou desde nível fundamental até pós-graduação. A renda familiar variou de 0,4 a 1,7 salários mínimos, per capita.

Quanto ao perfil das crianças e adolescentes, 4 eram do gênero feminino e 5 do gênero masculino. A média de idade das crianças e adolescentes com APLV, no momento da entrevista, foi de $7,1 \pm 3,4$ anos, variando de 2 anos a 12 anos. 0 tempo de dieta de exclusão foi, em média, de 6,4 $\pm 3,3$ anos, sendo que o tempo de acompanhamento no ambulatório de AA da Unidade de Alergia e Imunologia do ICr-HCFMUSP foi de 3,5 $\pm 2,3$ anos. A maioria apresentava outras doenças alérgicas e duas crianças também apresentavam reações a outros alimentos. Em relação ao tipo de reação alérgica ao LV, a maioria apresentava manifestações dermatológicas e respiratórias, sendo que 7 crianças apresentavam história de anafilaxia.

$\mathrm{Na}$ análise dos discursos, foram obtidas três categorias e oito subcategorias: tratamento e educação do paciente e familiares (experiências vividas, base do tratamento e como lidar com a doença), resolução da doença (expectativa e melhora gradativa), qualidade de vida (inclusão social, cotidiano familiar e custo dos alimentos).

\section{Tratamento e educação do paciente \\ e familiares: experiências vividas}

Quanto às experiências vividas, os familiares colocaram sua opinião sobre o tratamento, comentaram sobre o desconhecimento da doença e as vivências de reações alérgicas.

Em relação ao tratamento, os familiares comentaram sobre o início do tratamento, a aceitação do diagnóstico e as dificuldades que enfrentaram antes de iniciar o acompanhamento em nosso ambulatório de AA. Relataram que a vida ficou mais fácil após as orientações recebidas e que, por isso, estavam muito satisfeitos com o tratamento:

...no começo foi bem difícil, muito complicado, porque eu nunca tinha ouvido falar de alergia ao leite, até ele nascer. Então, quando ele nasceu, logo eu consegui vir para cá e foi aonde eu tive orientação sobre a alergia. (D8US1)

Ao iniciar o acompanhamento no serviço, conseguiram entender melhor sobre a doença, o que facilitou bastante os cuidados com a criança.

Também constatamos que, após o período de adaptação à doença, os familiares perceberam que a APLV não é tão grave quanto outras doenças.

Além dos aspectos positivos do acompanhamento relatados pelos familiares, também notamos algumas queixas, como: o impacto das informações transmitidas na primeira consulta, as insatisfações e as dúvidas.

No que se refere à primeira consulta, em geral, são transmitidas muitas informações, as quais podem ser marcantes na vida dos familiares e provocar sentimentos de ansiedade e receio com o tratamento.

Quanto às insatisfações, percebemos que, muitas vezes, o tratamento não atende às expectativas dos responsáveis. Em um dos discursos, a avó de um adolescente de 12 anos demonstra sua frustração, pois, na sua visão, as condutas são sempre mantidas e as consultas de rotina não parecem trazer vantagens ao paciente, exceto pela consulta com a nutricionista, em que são fornecidas novas orientações e receitas:

Hoje eu me questionei quando eu saí de casa. Eu perco dia de serviço... (...) Então eu fico questionando: "eu vou lá fazer o quê?" A nutricionista ainda me orienta, ainda me passa algumas coisas (...), me deu um livrinho de dieta alimentar. Então ainda eu vejo vantagem, mas vir aqui consultar... (D5US4)

Em relação às dúvidas, notamos que estão relacionadas ao diagnóstico, tratamento e história natural. Observamos a falta de entendimento em relação à doença, a necessidade de se descobrir a causa da APLV, questionamentos quanto à existência de outras modalidades de tratamento (por exemplo, vacinas) e quanto à determinação da idade de tolerância:

... eu não sei se um dia ela vai tomar leite. Eu adoro leite, então, todas as comidas que eu gosto muito, o mingau, ela não pode tomar, então, nessa hora eu fico pensando: "será se isso aí vai mudar?" Mas, é uma pergunta que eu não sei responder, eu não sei nem se os médicos podem me dizer: "Não, daqui a tantos anos ela vai tomar leite". (D6US12)

Apesar da confiança na equipe médica do hospital, os familiares demonstraram que existe grande desconhecimento de outros médicos e da população sobre a APLV.

Verificamos experiências ruins dos familiares com outros médicos, por receber prescrições de medicamentos contendo lactose ou por ter dificul- 
dade de atendimento no pronto-socorro devido a uma reação anafilática:

(...) Dessa vez, ele chegou ao pronto-socorro passando mal. A sorte que o médico foi muito legal comigo. Atenderam ele primeiro, porque ele chegou lá sem ar, roxo, com a boca roxa, todo vermelho, com o peito chiando. (...) Porque não é sempre que o pronto-socorro atende as crianças na frente, atende uma pessoa que está passando mal na frente. (...) Dessa vez eu tive sorte. Porque assim que eu cheguei, eu nem fiz a ficha dele, já o atenderam, já deram injeção, já puseram soro. (D5US8)

Também houve relatos de conflitos com o pai da criança, por culpar a mãe pelo desenvolvimento da doença, atribuído à herança genética e por não apoiar a dieta de exclusão.

No começo, eu tive dificuldade com o pai dele. Porque o pai dele também não aceitava. E até hoje ele fala que a culpa é minha. Diz que é meu sangue que é ruim. Então sempre tem aquelas palavras que muitas vezes ferem a gente. Você até perdoa, mas não consegue esquecer, fica aquilo lá na cabeça. (D4US9)

Uma das mães referiu que as pessoas de seu convívio não acreditaram que seu filho tinha a alergia e ofereceram LV contra suas ordens. Além disso, afirmaram para a mãe que ele não poderia ter APLV, pois consideravam que era uma "doença de ricos".

Algumas dessas pessoas que me criticaram viram na própria pele que não era, até por me desobedecer, foram fazer experiência com ele, sofreram as consequências. Lógico que eu fiquei muito triste, mas elas também aprenderam por ver que não era besteira, que era uma coisa séria. (D4US7)

Quanto às reações alérgicas vivenciadas, observamos falta de preparo de familiares e profissionais de saúde, pois não conseguiram realizar as medidas necessárias para o tratamento das reações.

... como eu passei um transtorno no avião, onde ela comeu uma migalha de pão e aconteceu uma anafilaxia e eu passei muito transtorno, porque, em cima, lá no alto, eu dando para o médico adrenalina e ele se negando a aplicar que fenergan ia resolver. Foi muito constrangedor para a gente porque a gente chorava muito e não podia fazer nada com ela. (D6US8)

\section{Tratamento e educação do paciente e familiares: base do tratamento}

Nesta subcategoria, os familiares apresentaram seus questionamentos sobre o que é tratamento. Percebemos que o termo tratamento pode ser relacionado a medicamento ou vacina, e que a dieta de exclusão seria considerada apenas uma orientação e não tratamento:

Porque até agora, eu não vi tratamento nenhum. Não tem! Eu venho aqui só receber orientação. (D5US10)

Verificamos o desejo de que a criança recebesse um medicamento ou vacina para curá-la da alergia, demonstrando uma relação direta entre tratamento e cura.
Também fica evidente a impotência de todos para acelerar o processo de tolerância, onde a ingestão do alimento não provocaria mais a doença. Desta forma, não há maneiras para a criança tornar-se tolerante mais rapidamente, sendo preciso ter paciência e esperar.

\section{Tratamento e educação do paciente e familiares: como lidar com a doença}

Os familiares ressaltaram a importância do aprendizado sobre como lidar com a APLV, por exemplo, alimentos permitidos e proibidos, leitura de rótulos, fornecimento de receitas pela nutricionista, e o planejamento das refeições. Além disso, também destacaram a importância de adesão ao tratamento, tanto por parte da criança, como do responsável e da escola.

Se eu não ler todos com sabedoria, corro o risco. Agora ele está bem, cem por cento bom, mas antes tinha que ler todos com muita cautela, porque senão, era assim, colocava ele em risco, primeiro estourava a pele, depois vinha o chiado e o vômito, vinha tudo. (D1US11)

Antes meu filho falava: "Você não faz um bolo pra mim mãe?" E hoje eu sei fazer tudo, bolo, preparar tanta coisa sem leite... (D1US24)

Apesar do esforço em seguir as orientações, uma grande dificuldade encontrada pelas mães, correspondeu ao controle da alimentação dos fiIhos pequenos, especialmente quando tinham irmãos, pois a criança queria consumir os mesmos alimentos que eles:

Porque ele não entende. Eu falo, às vezes, é muito rápido. Eu tenho uma menina de oito. Então, come as coisas escondido. Se ele ver um papel, ele já sabe. Ele quer aquilo. Eu falo: "Não Alexandre, você não pode comer isso". Aí ele grita, chora até vomita de tanto chorar. Então eu acho que agora, com essa idade dele, está mais difícil para eu controlar. Está muito difícil. (D3US5)

Contudo, conforme a criança cresce, passa a entender melhor sobre sua condição e também auxilia na adesão ao tratamento. Essa a conscientização da criança é um fator de tranquilidade para a mãe e facilita a adesão à dieta de exclusão.

Ao ingressar na escola, os professores e outros funcionários envolvidos têm papel fundamental na colaboração com o tratamento:

E a escolinha, porque ele fica particularmente $9 \mathrm{~h}$ na escola. Então a escola sabe o que ele pode e o que ele não pode. (D3US4)

Já os adolescentes, podem ter consciência da dieta de exclusão, mas não necessariamente aderem ao tratamento, o que pode causar preocupação aos familiares:

Ele sai de casa, ele vai para uma festa de aniversário, eu rezo (...) para ele. Olha: "não come bolo, (...) não come nada que tiver lá." O salgadinho, dependendo do salgadinho, se (...) tiver leite, ele passa mal. (D5US7)

Também percebemos que os pais podem encarar a dieta de exclusão de forma positiva, proporcionando alimentação mais saudável ou de forma negativa, encarando como um sofrimento para 
a criança, por não poder ingerir os alimentos que tem vontade:

Eu sei que tem muitos alimentos ou coisas, guloseimas, que ela não come, que para muitas mães: "Ah eu estou triste, ela vai para uma festinha e ela não come". Eu nem penso assim hoje, hoje eu penso que o que ela come é muito saudável, ela tem uma alimentação muito nutrida. (D6US4)

\section{Resolução da doença: expectativa}

Os familiares comentaram sobre o desejo de cura, no sentido de atingir a tolerância. Compreendemos que eles sabem que a chance de tolerância aumenta com a idade e que é dependente do próprio organismo da criança.

O que eu espero, daqui uns dois a três anos, até mais, não sei, depende muito do organismo dele, ele estará cem por cento bom... (D1US15)

As mães também descreveram claramente que é natural ter essa esperança, pois faz parte do papel de ser mãe, isto é, gostar dos filhos e querer o melhor para eles.

Além disso, constatamos que a esperança de cura faz com que as mães se mantenham firmes no acompanhamento, acreditando que a tolerância será atingida com o seguimento do tratamento.

\section{Resolução da doença: melhora gradativa}

Um dos aspectos positivos observados ocorre pela satisfação da diminuição do número de reações alérgicas.

... graças a Deus eu estou vendo uma evolução muito grande. Antigamente não podia nem relar nele que ele entrava em crise alérgica. Hoje em dia não, ele já consegue ir para a escola, ele toma lanche com todo mundo. Então eu estou vendo que está resolvendo. (D8US4)

Também notamos uma satisfação quanto à melhora gradativa, isto é, diminuição da gravidade dos sintomas, tolerância a traços de leite, possibilidade de ir à escola e fazer o lanche em companhia dos amigos.

Os familiares têm consciência de que existe uma evolução durante o tratamento, mas que é lenta e gradual. Assim como andar e falar são grandes conquistas comemoradas pelos pais, percebemos que o fato de poder consumir alimentos com traços de leite e fazer o lanche com amigos também são comemorados:

Agora ela já sabe, porque come de pouquinho, porque está começando. Tudo como se fosse a primeira vez, andar, falar, a ficar de pé. Agora está melhor. (D9US7)

Constatamos que a fé em Deus está bastante presente nas falas das mães e com isso, conseguem ter esperança e superar mais facilmente os problemas enfrentados no dia a dia:

... eu peço muito a Deus, em primeiro lugar, que ele melhore. Porque eu comecei aqui, ele iria fazer um ano... o tratamento, ele tinha 1 ano, eu acho. E eu estou aí, para o que der e vier, vamos ver. (D3US9)

\section{Qualidade de vida: inclusão social}

Os familiares comentaram sobre o esforço para que a criança viva normalmente, como qualquer outra criança, frequentando a escola, comendo os alimentos que deseja, sem exclusões.

... eu não deixo de medir esforços para que ela viva bem. (D6US11)

Além disso, o acompanhamento especializado proporciona apoio e orientações para que consigam melhorar suas vidas, mesmo que enfrentem situações indesejadas e com isso, os pais percebem que a criança pode viver bem.

Por mais que ela tenha muitas restrições, mas eu tentei adequar a vida dela de uma forma que ela se sinta melhor e sem nenhuma exclusão. O médico lá em minha cidade queria que eu não botasse ela para estudar e eu não pensei assim, eu botei, eu já tive um transtorno na escola, mas aqui vocês dão força: "vai, tem que botar ela para estudar, ela é normal". (D6US5)

\section{Qualidade de vida: cotidiano familiar}

Os familiares comentaram sobre a preocupação com os cuidados com a criança, o emprego e o medo de uma reação alérgica. O fato de deixar de trabalhar demonstra o sacrifício dos próprios interesses em função dos cuidados à criança. Já para quem trabalha e deixa a criança sozinha, é estressante pensar que pode acontecer algum imprevisto e não ser possível ajudar.

A Joana, na minha vida, são dois momentos, antes da Joana e depois da Joana. Depois que ela nasceu eu enfrentei muitas barreiras. E eu tenho uma vida muito dedicada a ela. Talvez esse ano foi o ano que eu comecei a trabalhar de volta mesmo, porque desde que ela nasceu com esse problema foi difícil. (D6US1)

Mas é o meu medo, em uma hora eu estar trabaIhando, ele estar sozinho, ele abusar, fazer alguma besteira e não ter ninguém para acudir. É meu medo. (D5US9)

\section{Qualidade de vida: custo dos alimentos}

Apenas uma mãe relatou em nosso estudo a dificuldade financeira para comprar os alimentos, a falta de produtos próximo de sua residência e o trabalho que tem para procurar esses alimentos.

...os alimentos são caros também, são bem caros e aonde eu moro é um pouco complicado para comprar também, porque tem supermercado que não tem. Então fica até um pouco difícil. O leite também é bem mais caro... (D7US4)

\section{DISCUSSÃO}

Este é o primeiro estudo qualitativo que tem como base a população os familiares de crianças e adolescentes brasileiros com APLV. Os resultados permitiram entender como os familiares enfrentam a doença, suas histórias e as expectativas quanto ao tratamento.

As subcategorias base do tratamento e meIhora gradativa não eram esperadas e não foram encontradas em outros estudos. No que se refere à base 
do tratamento, provavelmente esta subcategoria seja um reflexo do grau de compreensão dos familiares deste estudo. Talvez seja parte da cultura popular esta visão de tratamento relacionada a medicamento e seja difícil compreender que a dieta de exclusão também é uma modalidade de tratamento. Em relação à melhora gradativa, possivelmente esta subcategoria não foi encontrada em outros estudos devido à presença de AAs que têm como característica a persistência de sintomas graves, como alergias a amendoim e frutas oleaginosas.

Quanto às experiências vividas, observamos dificuldade de aceitação do diagnóstico de AA, assim como em outro estudo australiano7, sendo constatado um processo de adaptação ao longo do tempo de seguimento.

Notamos que os familiares ainda apresentam dúvidas em relação ao tratamento, mesmo após seguimento prolongado. Outros autores constataram que a maioria dos familiares de crianças com AA têm um desejo muito grande por informações no início do tratamento. ${ }^{7}$ Em vista disso, é importante estabelecer um bom vínculo do profissional de saúde com a família para criar uma confiança mútua e proporcionar a troca de informações nos dois sentidos, facilitando o cuidado bem sucedido do paciente ${ }^{17}$.

Percebemos que o fato de ter acompanhamento com a nutricionista é destacado como um diferencial da instituição, já que proporciona alternativas alimentares e facilita a instituição da dieta. Além disso, os familiares revelaram ser fundamental obedecer a cada orientação e que era um desperdício de tempo ir às consultas e não aderir ao tratamento. Portanto, verificamos que há satisfação com o tratamento fornecido pela instituição e confiança na equipe.

Entretanto, ainda há desconhecimento dos profissionais de saúde em geral e da população sobre AA, assim como constatado por outros pesquisadores $^{18}$. O maior conhecimento sobre a doença e seu tratamento proporcionará maior apoio, segurança e QV aos familiares e pacientes com APLV ${ }^{18}$.

As orientações sobre o tratamento devem ser práticas, para auxiliar no planejamento das refeições e facilitar a adesão ao tratamento. Uma das orientações fundamentais corresponde à leitura de rótulos, tarefa difícil, pela quantidade de termos sinônimos ao leite existentes, pelo tamanho das letras em rótulos de produtos e pela própria legislação existente no Brasil ${ }^{19}$.

Neste contexto, a atuação de nutricionista na equipe multiprofissional que lida com pacientes com APLV é de fundamental importância pela facilitação no entendimento de termos presentes nos rótulos, na pesquisa constante de variações nos constituintes de produtos de uso rotineiro e na atualização de listas de produtos que devem ser evitados, pela consulta junto aos serviços de atendimento ao consumidor (SACs) de cada indústria alimentícia. Sua função não se restringe apenas a fornecer receitas alternati- vas, mas a instruir os pacientes e familiares de maneira a incutir em suas rotinas todos os passos que se referem aos cuidados com a alimentação.

O suporte de todos aqueles que mantêm contato com o paciente é fundamental. Para ajudar as mães, principais cuidadoras, o atendimento e orientações podem ser estendidos aos demais familiares (pai, avós, tios) e outros cuidadores (professores, vizinhos, etc), sempre que possível, solicitando a presença na consulta ou disponibilizando materiais informativos.

É importante que os cuidadores sejam informados quanto à história natural da APLV e a aquisição de tolerância ao leite, percebendo que a doença pode não persistir pelo resto da vida e o tratamento poderá ser por período limitado. Neste aspecto, outros estudos demonstraram que a religião e a espiritualidade estavam presentes nas famílias de crianças com outras doenças crônicas, proporcionando sentimentos de conforto, esperança e suporte para a aceitação das condições impostas pela doença ${ }^{17}$.

Devido à constatação visível de melhora, não observamos relatos de esgotamento, cansaço ou desânimo, como em outros estudos de pacientes com doenças crônicas ${ }^{20}$, o que nos fortalece em continuar o trabalho multidisciplinar realizado em nosso serviço.

Em relação à inclusão social, é importante ressaltar que os familiares de nosso estudo se preocupam com a QV de seus filhos e se esforçam para que eles tenham uma vida normal, o que pode ter repercussões positivas para a criança.

Verificamos alterações na vida familiar, especialmente das mães, que deixam de trabalhar para cuidar da criança alérgica e dificuldades financeiras para aquisição de alimentos especiais.

Em estudo realizado na Nova Zelândia, os participantes também referiram aumento no custo de vida, incluindo consultas médicas, aquisição de adrenalina e dos alimentos isentos dos alérgenos ${ }^{21}$. Muitos participantes relataram necessitar procurar os alimentos necessários em diferentes supermercados. Os custos também foram citados pelos participantes de um estudo canadense ${ }^{6}$.

Apesar da crescente oferta de produtos alimentícios destinados a pacientes com APLV, o preço elevado ainda não permite que sejam acessíveis a populações de menor poder aquisitivo, o que pode acarretar piora na QV.

Assim, as experiências dos familiares em relação à APLV podem ser variáveis dependendo do cenário envolvido. Em geral, verificamos satisfação com o tratamento proporcionado pela instituição, mas há dificuldade com os demais profissionais de saúde, familiares e pessoas do convívio social, bem como problemas para lidar com a doença no dia a dia. Observamos dúvidas quanto à doença e falta de compreensão de que o tratamento da APLV não envolve uso de medicamentos diários. Apesar das limitações impostas pela doença, há expectativa de melhora e de cura, além de esforços para proporcionar uma boa QV. A melhor forma de podermos entender as perspectivas dos familia-

- 6 -res e pacientes com APLV é a possibilidade de ouvir 


\section{REFERÊNCIAS}

1. Cummings AJ, Knibb RC, King RM, Lucas JS. The psychosocial impact of food allergy and food hypersensitivity in children, adolescents and their families: a review. Allergy. 2010;65(8):933-45.

2. Teufel M, Biedermann T, Rapps N, Hausteiner $C$, Henningsen $P$, Enck $P$, et al. Psychological burden of food allergy. World J Gastroenterol. 2007;13(25):3456-65.

3. Bollinger ME, Dahlquist LM, Mudd K, Sonntag $C$, Dillinger L, McKenna K. The impact of food allergy on the daily activities of children and their families. Ann Allergy Asthma Immunol. 2006;96(3):415-21.

4. Mandell D, Curtis R, Gold M, Hardie S. Anaphylaxis: how do you live with it? Health Soc Work. 2005;30(4):325-35.

5. Akeson N, Worth A, Sheikh A. The psychosocial impact of anaphylaxis on young people and their parents. Clin Exp Allergy. 2007; 37(8): 1213-20.

6. Gillespie CA, Woodgate RL, Chalmers KI, Watson WT. "Living with risk": mothering a child with food-induced anaphylaxis. J Pediatr Nurs. 2007;22(1):30-42.

7. Hu W, Grbich C, Kemp A. Parental food allergy information needs: a qualitative study. Arch Dis Child. 2007;92(9):771-5.

8. Monks H, Gowland MH, MacKenzie H, ErlewynLajeunesse M, King R, Lucas JS, et al. How do teenagers manage their food allergies? Clin Exp Allergy. 2010;40(10):1533-40.

9. MacKenzie H, Roberts $G$, van Laar D, Dean $T$. Teenagers' experiences of living with food hypersensitivity: a qualitative study. Pediatr Allergy Immunol. 2010;21(4 Pt 1):595-602.

10. Polit DF, Beck CT, Hungler BP. Fundamentos de pesquisa em enfermagem. $5^{a}$ ed. Porto Alegre: Ed Artmed; 2004.
11. Bosi MLM, Mercado-Martínez F. Pesquisa qualitativa de serviços de saúde. Rio de Janeiro: Editora Vozes; 2004.

12. Meihy JCSB, Holanda F. História oral: como fazer, como pensar. São Paulo: Contexto; 2007.

13. Bardin L. Análise de conteúdo. Lisboa: Edições 70; 2000.

14. Campos CJG, Turato ER. Análise de conteúdo em pesquisas que utilizam metodologia clínico-qualitativa: aplicação e perspectivas. Rev Latino-Am Enfermagem. 2009;17(2):259-64.

15. Houaiss, A. Dicionário Houaiss da língua portuguesa. [acesso em 01 fev 2010]. Disponível em: http://biblioteca.uol.com.br/.

16. Michaelis. Moderno Dicionário da língua portuguesa. [acesso em 01 fev 2010]. Disponível em: http://biblioteca.uol.com.br/.

17. Edmonds-Myles S, Tamborlane WV, Grey M. Perception of the impact of type 1 diabetes on low-income families. Diabetes Educ. 2010;36(2):318-25.

18. Gupta RS, Kim JS, Barnathan JA, Amsden LB, Tummala LS, Holl JL. Food allergy knowledge, attitudes and beliefs: focus groups of parents, physicians and the general public. BMC Pediatr. 2008;19(8):36.

19. Binsfeld $B L$, Pastorino AC, Castro APBM, Yonamine $\mathrm{GH}$, Gushken AKF, Jacob CMA. Conhecimento da rotulagem de produtos industrializados por familiares de pacientes com alergia a leite de vaca. Rev Paul Pediatr. 2009; 27(3):296-302.

20. Damião EBC, Angelo M. A experiência da família ao conviver com a doença crônica da criança. Rev Esc Enf USP. 2001;35(1):66-71.

21. McBride $C$, McBride-Henry $K$, van Wissen $K$. Parenting a child with medically diagnosed severe food allergies in New Zealand: the experience of being unsupported in keeping their children healthy and safe. Contemp Nurse. 2010;35(1):77-87. 